\title{
EUS-guided gallbladder drainage in patients with cirrhosis: results of a multicenter retrospective study
}

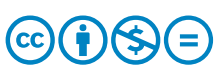

\author{
Authors \\ Institutions \\ 1 University of North Carolina, Division of \\ Gastroenterology \& Hepatology, Chapel Hill, North \\ Carolina, United States \\ 2 West Virginia Ruby Memorial Hospital Digestive \\ Diseases, Morgantown, West Virginia, United States \\ 3 University of North Carolina, Department of Medicine, \\ Chapel Hill, North Carolina, United States
}

Theodore W. James ${ }^{1}$, Matthew Krafft ${ }^{2}$, Michael Croglio ${ }^{3}$, John Nasr ${ }^{2}$, Todd Baron ${ }^{1}$

submitted 25.3.2019

accepted after revision 12.6.2019

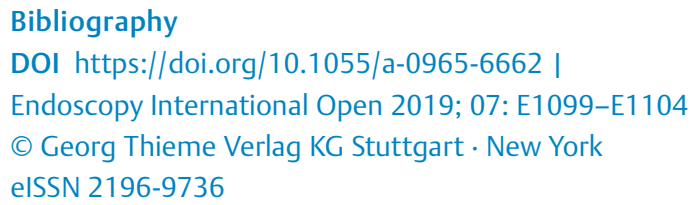

Corresponding author

Theodore W. James, University of North Carolina, Division of Gastroenterology \& Hepatology, 101 Manning Drive, Chapel Hill, NC 27514

Ted@unc.edu

\section{ABSTRACT}

Background and study aims Cirrhosis has historically been considered a relative, if not absolute, contraindication to cholecystectomy. Endoscopic ultrasound-guided gallbladder drainage (EUS-GBD) has been developed for use in non-operative candidates with cholecystitis; however, little data exist for use of the procedure in patients with cirrhosis.
Patients and methods This was a retrospective series involving two large tertiary referral centers performing EUSGBD. Patients with cirrhosis who underwent EUS-GBD for cholecystitis between August 2014 andDecember 2018 were identified. The primary endpoint was the rate of technical success, defined as EUS-guided placement of a lumenapposing metal stent (LAMS) from duodenum to gallbladder. Patient demographics, procedural details, adverse events (AEs), post-procedural symptoms, and clinical success were recorded.

Results Fifteen patients ( 9 females, 6 males) with cirrhosis underwent EUS-GBD during the study period. Mean patient age was $61 \pm 17.1 \mathrm{yrs}$, mean MELD-Na $15 \pm 7$. Etiology of cirrhosis was HCV $(n=2)$, alcohol $(n=4)$, non-alcoholic fatty liver disease $(n=8)$, and autoimmune hepatitis $(n=1)$.

The technical success rate was $93.3 \%$ and mean procedure time was $64 \pm 59$ minutes. Initial puncture site was duodenum $(n=11)$, stomach $(n=3)$ and jejunum $(n=1)$ and portion of gallbladder used for drainage was neck $(n=4)$ and body $(n=11)$. Fourteen patients went on to clinical success and two AEs occurred in this cohort. One decompensation event occurred in a patient with Child-Pugh class $C$ disease 3 weeks post-procedure. Mean length of follow-up was 373 \pm 367.3 days; one death occurred due to underlying malignancy.

Conclusion EUS-GBD is safe and efficacious in managing cholecystitis in patients with Child-Pugh A and B cirrhosis who are non-operative candidates. Further studies are needed to determine optimal patient selection and procedural technique.

\section{Introduction}

Acute cholecystitis is a common medical condition, conventionally treated with cholecystectomy, either open or laparoscopic [1]. Nearly 700,000 cholecystectomies are performed annually in the United States, making it one of the most common surgeries performed [2], however, many patients with cholecystitis may be poor operative candidates due to comor- bid conditions that increase risk for perioperative morbidity and mortality [3].

Percutaneous transhepatic gallbladder drainage (PTGBD) has demonstrated efficacy in temporary decompression of the gallbladder [4-6] PTGBD is limited by presence of severe coagulopathy, anatomically inaccessible gallbladders, and is associated with adverse events (AEs) including catheter dislodgment, cellulitis, pneumothorax, bleeding, and infection $[7,8]$. 
In addition, there is a high recurrence rate of cholecystitis if the catheter is removed [9].

Endoscopic gallbladder drainage was developed to supplant the need for PTGBD in treatment of cholecystitis in patients deemed unfit for surgery due to comorbidities or advanced malignancy unable to undergo cholecystectomy [10]. Endoscopic options include endoscopic retrograde cholangiopancreatography (ERCP) with transpapillary placement of a plastic stent into the cystic duct and endoscopic ultrasound-directed gallbladder drainage (EUS-GBD) [11]. ERCP and EUS-GBD have demonstrated similar efficacy, however, EUS-GBD has a higher technical success rate, particularly when the gallbladder is distended or the cystic duct is inaccessible due to tortuosity or obstruction [12].

Cirrhosis has historically been considered a relative, if not absolute, contraindication to laparoscopic cholecystectomy [3]. Gallstone prevalence in patients with cirrhosis ranges from $25 \%$ to $30 \%$, being at least twice that of the general population with a higher incidence in decompensated cirrhosis, irrespective of etiology [13]. Untreated, cholecystitis with or without gallstone disease can lead to repeat admissions for pain, or may lead to gallbladder perforation and death [14]. Sparse data exist on use of EUS-GBD in management of cholecystitis in patients with cirrhosis. The current study aimed to describe the outcome of EUS-GBD for cholecystitis in patients with cirrhosis.

\section{Patients and methods}

This retrospective series involved two large tertiary referral centers with two therapeutic endoscopists (THB and JN) performing EUS-GBD. The institutional review board at each center approved this study. All consecutive adult patients (age $\geq 18 \mathrm{yr}$ ) who underwent EUS-GBD for cholecystitis between August 1, 2014 and December 31, 2018 were identified using the electronic medical record. Patients without documented cirrhosis were excluded from analysis. Clinical and procedural data were collected, including etiology of cirrhosis, Child-Pugh score at time of procedure, model for end-stage liver disease sodium (MELD-Na) score at time of procedure, indication for EUS-GBD, endoscopic data (length and diameter of stent, anastomotic location, procedural findings), procedure-related AEs, post-procedural symptoms, and clinical success, when available. The primary endpoint was the rate of technical success, defined as EUS-guided placement of a lumen-apposing metal stent (LAMS) from the gastrointestinal lumen to the gallbladder. AEs were graded according to the American Society for Gastrointestinal Endoscopy lexicon [15]. Hepatic decompensation within 30 days following the procedure was defined according to the European Association for the Study of the Liver guidelines [16]. Sepsis was defined according to the Society of Critical Care Medicine [17]. Clinical success was defined as relief of symptoms of cholecystitis.

Statistical analyses were performed using Stata version 15.1 (StataCorp, Texas, United States). All continuous variables are expressed as mean \pm standard deviation and categorical variables are expressed as proportions (\%). Because of a small num-

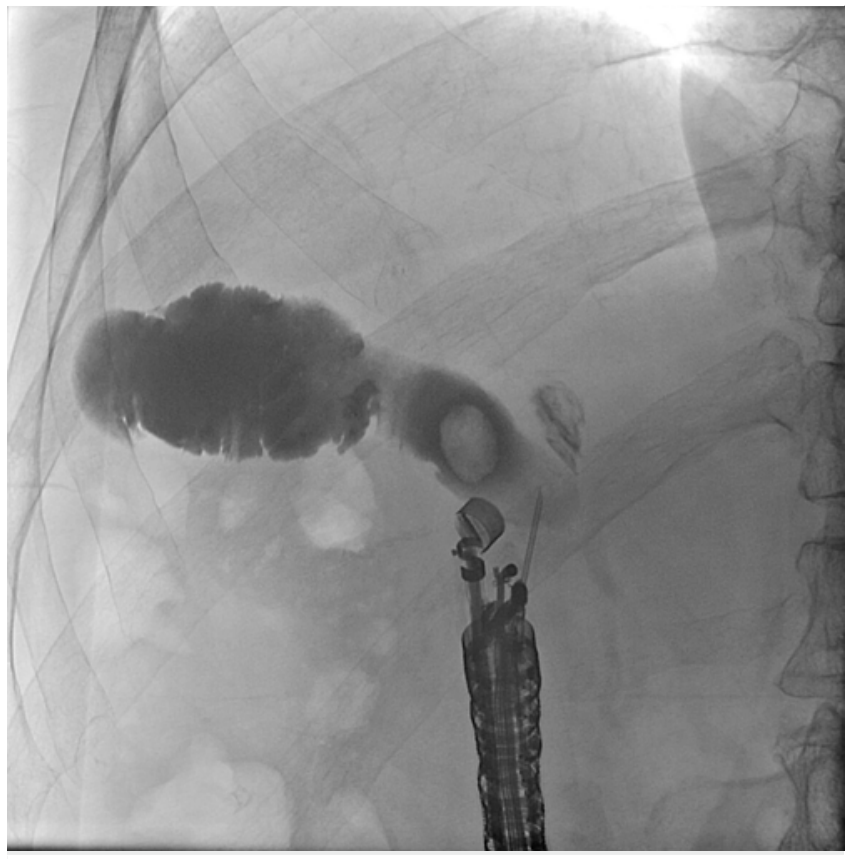

- Fig. 1 EUS-guided needle placement into the gallbladder confirmed with contrast injection under fluoroscopy.

ber of events, we did not perform a logistic regression analysis. $P<0.05$ was considered statistically significant. Neither sample size nor statistical power was calculated as this was not a comparative study.

Prior to undertaking the procedure, all patients were informed of the risks, benefits, and alternatives of proceeding with EUS-GBD and each provided written informed consent. General anesthesia and fluoroscopy were used in all cases. Patients with acute cholecystitis were continued on their antibiotic therapy; in the absence of infection, pre-procedural antibiotics were not routinely administered. The technique of EUSGBD was performed as follows: a standard therapeutic channel oblique linear echoendoscope (GF-UCT180, Olympus America, Center Valley, Pennsylvania, United States) was passed into the duodenum, stomach (3 cases) or jejunum (1 case) to visualize the gallbladder. Interposing vessels were identified by Doppler so that they could be avoided. One of two approaches was used. In the first, a $19 \mathrm{G}$ needle (Expect, Boston Scientific, Marlborough, Massachusetts, United States) preloaded with watersoluble contrast was used to puncture the gastrointestinal lumen into the gallbladder and entry was confirmed by contrast injection ( $\triangleright$ Fig. 1). The needle was flushed with saline and a 0.025-inch, 450-cm hydrophilic-tipped guidewire (VisiGlide, Olympus) was passed into the gallbladder ( $\mathbf{F i g . 2 )}$. The needle was withdrawn and the LAMS (AXIOS-EC, Boston Scientific, Marlborough, Massachusetts, United States) and electrocautery device was introduced through the working channel. Current was applied to the cautery tip and advanced into the gallbladder over the guidewire, with the distal portion deployed into the gallbladder and proximal into the gastrointestinal lumen ( $\triangleright$ Fig.3). In the latter cases and when the gallbladder was distended, the electrocautery-enhanced LAMS was passed 


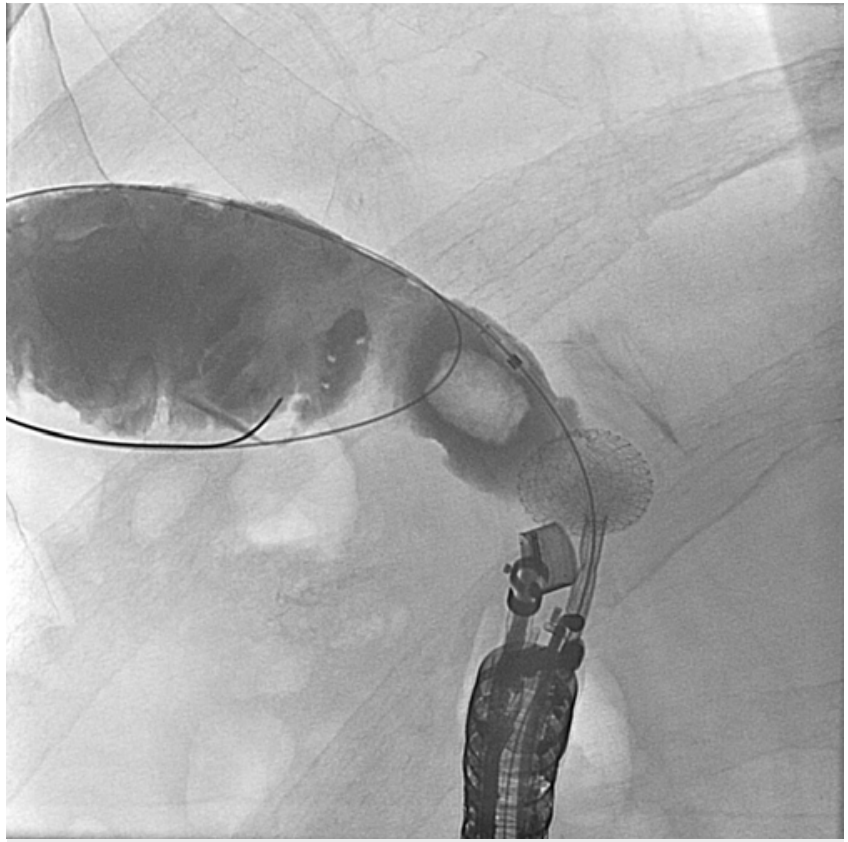

- Fig. 2 Guidewire passed into gallbladder in order to secure position and aid stent placement.

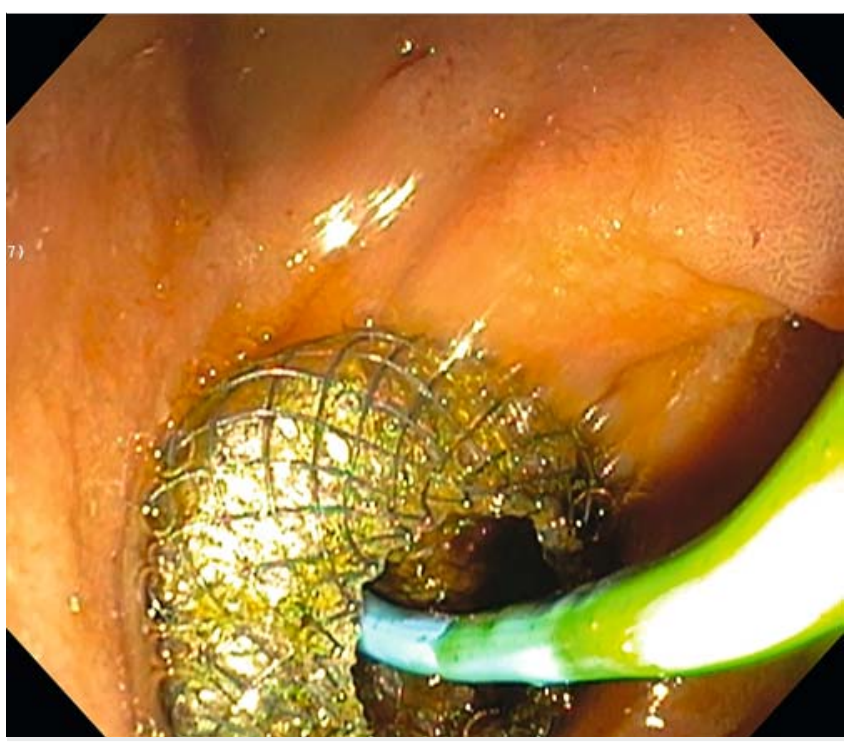

- Fig. 3 Endoscopic view of duodenum following cholecystoduodenostomy with lumen apposing metal stent and plastic pigtail stent placement within.

directly into the gallbladder without a guidewire. In all technically successful cases performed by one endoscopist (THB) a $7 \mathrm{Fr}$ double pigtail plastic stent was placed within the LAMS over a guidewire.

\section{Results}

A total of 15 patients (UNC:12, WVU:3, 9 females, 6 males) met inclusion criteria and underwent EUS-GBD during the study period. Mean patient age was $61 \pm 17.1$ years, mean MELD-Na
- Table 1 Patient demographics.

EUS-GBD patients with cirrhosis $(n=15)$

\begin{tabular}{|c|c|c|}
\hline Age, mean \pm SD & 61 & 17.1 \\
\hline Number of females (\%) & 9 & 60 \\
\hline \multicolumn{3}{|l|}{ Etiology of cirrhosis } \\
\hline Chronic Hepatitis C virus, n (\%) & 2 & 13 \\
\hline Alcohol, n (\%) & 4 & 27 \\
\hline Non-alcoholic fatty liver disease, n (\%) & 8 & 53 \\
\hline Autoimmune, n (\%) & 1 & 7 \\
\hline \multicolumn{3}{|l|}{ Child-Pugh class } \\
\hline A & 3 & 20 \\
\hline B & 10 & 67 \\
\hline C & 2 & 13 \\
\hline \multicolumn{3}{|l|}{ Ascites grade at time of procedure } \\
\hline 0 & 5 & 33 \\
\hline 1 & 7 & 47 \\
\hline 2 & 3 & 20 \\
\hline 3 & 0 & 0 \\
\hline \multicolumn{3}{|l|}{ INR at time of procedure } \\
\hline$<1.5$ & 8 & 53 \\
\hline $1.5-2.5$ & 7 & 47 \\
\hline MELD-Na, mean \pm SD & 15 & 7 \\
\hline \multicolumn{3}{|l|}{ Indication for EUS-GBD } \\
\hline Acute cholecystitis, n (\%) & 11 & 73 \\
\hline Chronic cholecystitis, n (\%) & 2 & 13 \\
\hline Recurrent gallstone pancreatitis, n (\%) & 2 & 13 \\
\hline Surgical consultation for cholecystectomy, n (\%) & 9 & 60 \\
\hline Indwelling percutaneous cholecystostomy tube, n (\%) & 3 & 20 \\
\hline
\end{tabular}

$15 \pm 7$, and Child-Pugh classes were $A(n=3), B(n=10)$ and $C$ $(n=2)(\triangleright$ Table 1). Etiology of cirrhosis was HCV $(n=2)$, alcohol $(n=4)$, non-alcoholic fatty liver disease $(n=8)$, and autoimmune hepatitis $(n=1)$. Indications for EUS-GBD included acute cholecystitis $(n=11)$, chronic cholecystitis $(n=2)$, and recurrent gallstone pancreatitis $(n=2)$. Nine patients had undergone surgical evaluation and been deemed to have a prohibitively high risk for perioperative morbidity and mortality. Three patients had previously had a percutaneous drain placed and EUS-GBD was being performed for gallbladder drain internalization. Three patients were septic at the time of the procedure.

The technical success rate for EUS-GBD was $93.3 \%(n=14)$ ( $\triangleright$ Table 2). Mean procedure time was $64 \pm 59$ minutes and initial puncture site was duodenum $(n=11)$, stomach $(n=3)$ and 
- Table 2 Procedural and outcomes data.

\begin{tabular}{|c|c|c|}
\hline \multicolumn{3}{|l|}{ EUS-GBD in patients with cirrhosis $(n=15)$} \\
\hline Technical success, n (\%) & 14 & 93.3 \\
\hline Procedure time, mean $\pm S D$, minutes & 64 & 59 \\
\hline \multicolumn{3}{|l|}{ Initial puncture site } \\
\hline Duodenum & 11 & \\
\hline Stomach & 3 & \\
\hline Jejunum & 1 & \\
\hline \multicolumn{3}{|l|}{ Portion of gallbladder used } \\
\hline Neck & 4 & \\
\hline Body & 11 & \\
\hline Gallbladder wall thickness, mean \pm SD, mm & 7 & 3.6 \\
\hline \multicolumn{3}{|l|}{ Stent diameters } \\
\hline $10 \mathrm{~mm}$ & 12 & \\
\hline $15 \mathrm{~mm}$ & 3 & \\
\hline Coaxial plastic stent placement, n (\%) & 9 & 60 \\
\hline \multicolumn{3}{|l|}{ Clinical outcome } \\
\hline Outpatient cases, n (\%) & 3 & 20 \\
\hline $\begin{array}{l}\text { Length of hospital stay post- procedure, } \\
\text { mean } \pm S D \text {, days }\end{array}$ & 2.9 & 3.9 \\
\hline \multicolumn{3}{|l|}{ Adverse events (severity grade) } \\
\hline Postprocedural pancreatitis, mild & 1 & 6.7 \\
\hline Stent misdeploy requiring surgery, severe & 1 & 6.7 \\
\hline $\begin{array}{l}\text { Patients with hepatic decompensation } \\
\text { following EUS-GBD, } \mathrm{n}(\%)\end{array}$ & 1 & 6.7 \\
\hline $\begin{array}{l}\text { Eventual EUS-GBD stent exchange with plastic } \\
\text { stent, } \mathrm{n}(\%)\end{array}$ & 7 & 46.6 \\
\hline $\begin{array}{l}\text { Length of time stent left in place prior to } \\
\text { exchange, mean } \pm S D \text {, days }\end{array}$ & 179 & 230 \\
\hline $\begin{array}{l}\text { Patients who went on to orthotopic liver } \\
\text { transplant, } \mathrm{n}(\%)\end{array}$ & 1 & 6.7 \\
\hline Length of follow-up, median (IQR) & 174 & 58,674 \\
\hline Patients with clinical success, $\mathrm{n}(\%)$ & 14 & 93.0 \\
\hline Deaths during follow-up period, n (\%) & 1 & 6.7 \\
\hline
\end{tabular}

jejunum ( $n=1)$ and portion of gallbladder used for drainage was neck $(n=4)$ and body $(n=11)$. Mean gallbladder wall thickness was $7 \pm 3.6 \mathrm{~mm}$ and the gallbladder was distended in all cases except one (93.3\%). EUS-GBD was performed using a LAMS in 14 patients and a non-foreshortening self-expandable metal stent (Viabil, W.L. Gore, Flagstaff, Arizona, United States) in one. Stent lengths were $10 \mathrm{~mm}(\mathrm{n}=14)$ and $40 \mathrm{~mm}(\mathrm{n}=1)$ with diameters of $10 \mathrm{~mm}(n=12)$ or $15 \mathrm{~mm}(n=3)$. In one case, a second overlapping stent of $40 \mathrm{~mm} \times 10 \mathrm{~mm}$ was required to bridge the distance between gallbladder and gastrointestinal lumen. In nine cases (60\%), a plastic pigtail stent was placed within the metal stent to decrease risk for stent occlusion; plastic stents were lengths of $3 \mathrm{~cm}(n=1), 4 \mathrm{~cm}(n=7)$ or $7 \mathrm{~cm}(n=1)$ with diameters of 7 French $(n=8)$ or 10 French $(n=1)$. In the two patients with recurrent gallstone pancreatitis, one patient had a pre-procedural magnetic resonance cholangiopancreatography demonstrating no stones in the common bile duct and the other patient had an ERCP with removal of choledocholithiasis prior to EUS-GBD.

The EUS-GBD stent was endoscopically removed in seven patients (46.6\%) after a mean of $179 \pm 230$ days; cholecystitis did not recur in any of these patients. No patients later underwent cholecystectomy, as their operative candidacy did not improve. One patient went on to receive an orthotopic liver transplant; $[18,19]$ operative documentation noted increased surgical complexity due to the dense scarring of the porta hepatis with the duodenum adherent to the gallbladder fossa, making dissection extremely challenging due to deformed anatomy and scar tissue. The duodenotomy created by the EUS-GBD was oversewn with gastric decompression via nasogastric tube and total parenteral nutrition. Eight patients did not have their stents removed and EUS-GBD served as destination therapy. Fourteen patients (93.3\%) went on to achieve clinical success, with relief of symptoms of cholecystitis.

Two AEs occurred in this cohort (13.3\%). One patient developed pancreatitis 1 week after stent placement and was managed supportively; this was considered a mild AE. In a second patient, the LAMS was maldeployed with the distal end in the gallbladder and the proximal end between the gastric wall and gallbladder; this was managed surgically with an ultimate favorable clinical outcome, but was considered a severe AE. One patient with Child-Pugh class C cirrhosis was admitted 3 weeks after the procedure for hepatic decompensation including gastrointestinal hemorrhage, hepatic encephalopathy, and worsening ascites. Mean length of follow-up post-procedure was $373 \pm$ 367.3 days. One death $(6.6 \%)$ occurred due to underlying malignancy in a patient with stage IV pancreatic ductal adenocarcinoma; this occurred 37 days following the procedure after the patient was on home hospice care.

\section{Discussion}

Patients with cirrhosis and symptomatic gallbladder disease offer unique challenges and require a tailored approach to therapy. This group of patients is more likely to develop surgical complications including bleeding due to impaired coagulation, infection due to compromised immune function, and poor wound healing due to malnutrition and/or ascites [20]. Cholecystectomy is a particularly high-risk surgery in this patient population, particular in patients with Child-Pugh Class C cirrhosis, hypoalbuminemia, and portal hypertension [21] Management of cirrhotic patients with acute or chronic cholecystitis has largely employed percutaneous drainage for gallbladder decompression. For most patients in this demographic, the drain is often destination therapy, without plans for cholecystectomy at a later date as their operative candidacy does not 
improve over time. Percutaneous gallbladder drains carry a risk of dislodgement, which may be increased by ascites or hepatic encephalopathy. Another option for management of cholecystitis in cirrhotic patients is transpapillary cystic duct placement via ERCP [22]. This procedure may be challenging due to acute angulation in the cystic duct or obstruction from cholecystolithiasis. In addition, long-term drainage is a concern due to the narrow diameter of the plastic stent used. A third nonsurgical option for management of gallbladder disease has arisen in recent years in the form of EUS-GBD, however, little data exist on its use in patients with cirrhosis.

The current study was a multicenter, retrospective analysis of consecutive patients with cirrhosis who underwent EUSGBD for management of non-operative gallbladder disease. This was a heterogeneous group with a wide age range (3391 years) and diverse underlying etiologies for liver disease. The MELD-Na score was a mean of 15, which correlates in most studies with an estimated 3-month mortality of $6.0 \%$ [23]. The mortality rate in our cohort (6.6\%) approximates this estimate with one patient death during follow up; however, the cause of death was not directly related to cirrhosis, but rather, metastatic pancreatic cancer.

In our cohort, one patient with cirrhosis due to autoimmune hepatitis underwent EUS-GBD for management of acute cholecystitis and later went on to receive an orthotopic liver transplant. On review of pre-procedural imaging, ERCP with transpapillary cystic duct stenting was not felt to be possible and PTGBD was limited by hepatic encephalopathy with concern for high risk of drain dislodgement. Though EUS-GBD was felt to be the best option at the time of the patient's critical illness from acute cholecystitis, we feel that in the majority of cases, EUS-GBD should not be used in patients being considered for liver transplantation. The added surgical complexity from gallbladder scarring and duodenotomy may prohibit the patient from undergoing the life-saving operation, an outcome that no endoscopist would wish for their patient if it can be prevented. The decision to perform EUS-GBD should be made in a multidisciplinary fashion, with agreement from the transplant surgery team when applicable.

Long-term placement of an expandable metal stent into the gallbladder is not advisable as there are concerns for delayed bleeding and perforation. In addition, removal after a prolonged indwelling period may not be possible due to degradation of the stent covering; this could hypothetically lead to obstruction from tissue hyperplasia. However, there is currently no agreed upon duration to leave an expandable metal stent in place following EUS-GBD. Our typical approach to stent exchange is similar to the strategy employed in walled-off pancreatic necrosis drainage. In most cases of EUS-GBD, we electively replace the stent after tract maturation (2-4 weeks) with plastic pigtail stents to allow for continuous drainage without concern for recurrent cholecystitis. This plastic stent can be placed in most circumstances with a forward-viewing upper endoscope and provides an opportunity to assess the fistulous tract and extract accessible gallstones if present.

Limitations of the current study include retrospective design and relatively limited sample size. In addition, few patients with advanced cirrhosis, as determined by Child-Pugh Class, were included in this study. This is likely due to patient selection factors including increased risk for post-procedural bleeding, technical complexity of stent placement with comorbid ascites, and risk for infection due to impaired immune function. Future studies should be performed in a prospective fashion, ideally in a randomized controlled trial against either PTGBD or ERCP with transpapillary cystic duct stent placement.

\section{Conclusion}

In summary, the current study shows the feasibility of using EUS-GBD in patients with cirrhosis and gallbladder disease who are deemed nonsurgical candidates due to perioperative risk. This approach, using a LAMS, is safe and efficacious in management of gallbladder disease in this patient population. We believe such an approach will be preferred over percutaneous therapy, however, further studies are needed to determine optimal patient selection and procedural technique. As the growing burden of non-alcoholic fatty liver disease leads to an increasing patient population with comorbid cirrhosis and gallstone disease, further innovation is needed to effectively care for these patients.

\section{Competing interests}

Dr. Baron is a consultant and speaker for Boston Scientific, W. L. Gore, Cook Endoscopy, and Olympus America.

\section{References}

[1] Duncan CB, Riall TS. Evidence-based current surgical practice: calculous gallbladder disease. J Gastrointest Surg 2012; 16: 2011 - 2025

[2] Csikesz NG, Tseng JF, Shah SA. Trends in surgical management for acute cholecystitis. Surgery 2008; 144: 283-289

[3] Agresta F, Campanile FC, Vettoretto $\mathrm{N}$ et al. Laparoscopic cholecystectomy: consensus conference-based guidelines. Langenbeck's Arch Surgery 2015; 400: 429-453

[4] Chopra S, Dodd GD III , Mumbower AL et al. Treatment of acute cholecystitis in non-critically ill patients at high surgical risk: comparison of clinical outcomes after gallbladder aspiration and after percutaneous cholecystostomy. Am J Roentgenol 2001; 176: 1025-1031

[5] Ito K, Fujita N, Noda Y et al. Percutaneous cholecystostomy versus gallbladder aspiration for acute cholecystitis: a prospective randomized controlled trial. Am J Roentgenol 2004; 183: 193-196

[6] Patel M, Miedema BW, James MA et al. Percutaneous cholecystostomy is an effective treatment for high-risk patients with acute cholecystitis. Am Surgeon 2000; 66: 33

[7] Itoi T, Sofuni A, Itokawa F et al. Endoscopic transpapillary gallbladder drainage in patients with acute cholecystitis in whom percutaneous transhepatic approach is contraindicated or anatomically impossible (with video). Gastrointest Endosc 2008; 68: 455-460

[8] Oku T, Horii T, Masaka T et al. Clinical comparison of endoscopic naso-gallbladder drainage versus percutaneous transhepatic gallbladder drainage for acute cholecystitis. Nihon Shokakibyo Gakkai Zasshi 2013; 110: 989 - 997 
[9] Granlund A, Karlson BM, Elvin A et al. Ultrasound-guided percutaneous cholecystostomy in high-risk surgical patients. Langenbeck's Arch Surg 2001; 386: $212-217$

[10] Baron TH, Topazian MD. Endoscopic transduodenal drainage of the gallbladder: implications for endoluminal treatment of gallbladder disease. Gastrointest Endosc 2007; 65: 735 - 737

[11] Itoi T, Coelho-Prabhu N, Baron TH. Endoscopic gallbladder drainage for management of acute cholecystitis. Gastrointest Endosc 2010; 71: $1038-1045$

[12] Park JK, Woo YS, Noh DH et al. Efficacy of EUS-guided and ERCPguided biliary drainage for malignant biliary obstruction: prospective randomized controlled study. Gastrointest Endosc 2018: doi:10.1016/j.gie.2018.03.015

[13] Harvey VS. Incidence of cholelithiasis among patients with cirrhosis and portal hypertension. Gastrointest Radiol 1988; 13: 347-350

[14] Shi X, Jin S, Wang S et al. Gallbladder perforation in a patient with alcoholic liver cirrhosis and asymptomatic gallstones: A case report. Medicine 2018; 97: e0414

[15] Cotton PB, Eisen GM, Aabakken L et al. A lexicon for endoscopic adverse events: report of an ASGE workshop. Gastrointest Endosc 2010; 3: $446-454$

[16] Angeli P, Bernardi M, Villanueva C et al. EASL Clinical Practice Guidelines for the management of patients with decompensated cirrhosis. J Hepatol 2018; 69: 406 - 460
[17] Singer M, Deutschman CS, Seymour CW et al. The third international consensus definitions for sepsis and septic shock (Sepsis-3). Jama 2016; 315: 801 - 10

[18] Baron TH, Grimm IS, Gerber DA. Liver transplantation after endoscopic ultrasound-guided cholecystoduodenostomy for acute cholecystitis: a note of caution. Liver Transplant 2015; 21: 1322-1323

[19] Baron TH, Zacks S, Grimm IS. Endoscopic ultrasound-guided cholecystoduodenostomy for acute cholecystitis in a patient with thrombocytopenia and end-stage liver disease awaiting transplantation. Clin Gastroenterol Hepatol 2015; 13: e13-14

[20] Friedman LS. The risk of surgery in patients with liver disease. Hepatology 1999; 29: $1617-1623$

[21] Curro G, lapichino G, Melita G et al. Laparoscopic cholecystectomy in Child-Pugh class C cirrhotic patients. J Soc Laparoendosc Surgeons 2005; 9: 311

[22] Tujios SR, Rahnama-Moghadam S, Elmunzer JB et al. Transpapillary gallbladder stents can stabilize or improve decompensated cirrhosis in patients awaiting liver transplantation. Journal of clinical gastroenterology 2015; 49: $771-777$

[23] Wiesner R, Edwards E, Freeman R et al. Model for end-stage liver disease (MELD) and allocation of donor livers. Gastroenterology 2003; 124: $91-96$ 